\title{
3D Printing Microactuators for Soft Microrobots
}

\author{
Manav Tyagi, ${ }^{1,2}$ Geoffrey M. Spinks, ${ }^{2}$ and Edwin W.H. Jager ${ }^{1,2}$
}

\begin{abstract}
Current additive manufacturing, including three-dimensional (3D) and so-called four-dimensional printing, of soft robotic devices is limited to millimeter sizes. In this study, we present additive manufacturing of soft microactuators and microrobots to fabricate even smaller structures in the micrometer domain. Using a custom-built extrusion 3D printer, microactuators are scaled down to a size of $300 \times 1000 \mu \mathrm{m}^{2}$, with minimum thickness of $20 \mu \mathrm{m}$. Microactuators combined with printed body and electroactive polymers to drive the actuators are fabricated from computer-aided design model of the device structure. To demonstrate the ease and versatility of 3D printing process, microactuators with varying lengths ranging from 1000 to $5000 \mu \mathrm{m}$ are fabricated and operated. Likewise, microrobotic devices consisting of a rigid body and individually controlled free-moving arms or legs are 3D printed to explore the microfabrication of soft grippers, manipulators, or microrobots through simple additive manufacturing technique.
\end{abstract}

Keywords: 3D printing, microactuators, microrobots, soft robotics

\section{Introduction}

W ITH THE ADVENT OF ELECTROACTIVE POLYMER (EAP) technology and the potential to impact various fields of science, the need to develop or improve fabrication methodologies for EAP devices is of utmost importance. A particularly difficult challenge occurs when scaling down EAP actuators for use in miniaturized robotic devices. Complicated processing of EAP actuator devices based on microfabrication techniques such as photolithography makes it difficult for its integration with the current market or technologies. Additive manufacturing offers numerous advantages over the conventional technologies, including easier modification during product development due to simpler design to product process, saving on energy costs, and reducing waste production. Three-dimensional (3D) printing could certainly simplify the manufacturing of polymer actuators, making these devices potentially cheaper and available for a larger range of applications.

Soft actuators or robots have been $3 \mathrm{D}$ printed using a range of different materials and approaches. ${ }^{1,2}$ For example, soft dielectric elastomers were 3D printed using hybrid dielec- tric materials to fabricate a unimorph, ${ }^{3}$ electrically driven actuator. ${ }^{4}$ Although easy to fabricate using the additive manufacturing techniques, these actuators were $3 \mathrm{D}$ printed in the centimeter scale with minimum thickness of $200 \mu \mathrm{m}$, which means that they require very high actuation potentials (4-5 kV) for the movement. Using 3D printing, soft robotic actuators based on so-called McKibben muscles, integrated with bioinspired architectures, were fabricated to achieve programmable motions with a printing accuracy until $300 \mu \mathrm{m} .{ }^{5}$ Somatosensitive soft actuators, fabricated using embedded 3D printing, have been demonstrated to be used as robotic grippers that can provide proprioceptive and haptic feedback. ${ }^{6}$ Shape memory polymers have also been $3 \mathrm{D}$ printed to fabricate fast-responsive soft actuators with tunable mechanical properties allowing for flexibility and adaptability. ${ }^{7}$ These soft actuators were fabricated using hybrid multimaterial 3D printing down to $800 \mu \mathrm{m}$. Ionic polymer-metal composites have also been printed to fabricate soft actuators through fused-filament additive manufacturing. Using the technique, soft robotic devices with variety of shapes and sizes in the millimeter scale were 3D printed, with a thickness of at least $1000 \mu \mathrm{m} .{ }^{8,9}$ The field of soft robotics is

\footnotetext{
${ }^{1}$ Sensor and Actuator Systems, Department of Physics, Chemistry, and Biology (IFM), Linköping University, Linköping, Sweden.

${ }^{2}$ Australian Institute of Innovative Materials, Intelligent Polymer Research Institute, ARC Centre of Excellence for Electromaterials Science, University of Wollongong, Wollongong, Australia.

(C) Manav Tyagi et al. 2020; Published by Mary Ann Liebert, Inc. This Open Access article is distributed under the terms of the Creative Commons Attribution Noncommercial License (http://creativecommons.org/licenses/by-nc/4.0/) which permits any noncommercial use, distribution, and reproduction in any medium, provided the original author(s) and the source are cited.
} 
approaching the submillimeter domain, and there is a need to develop the additive manufacturing methods to microfabricate soft actuators at micrometer scales.

In recent years, the technique of additive manufacturing in $3 \mathrm{D}$ has been extended to transformable materials, which change their shape or dimension with time through some external stimulus, sometimes addressed as four-dimensional (4D) printing. ${ }^{10,11}$ The technique has also been implemented to fabricate polymer based soft actuators; for example, actuators based on stimuli responsive elastomers for softrobotic applications and magnetic soft actuators showcasing biomimicry were fabricated through 4D printing technique. $^{12,13}$ Similarly, hydrogel based, shape morphing structures were $4 \mathrm{D}$ printed to produce reversible deformations for soft actuation applications. ${ }^{14,15}$ However, these 3D/4D printing techniques were limited to millimeter sizes only, and to extend the scalability of this process, we are demonstrating the use of $3 \mathrm{D}$ printing process to fabricate even smaller structures in the micrometer domain.

Scaling down soft actuators based on conducting polymers (CPs; EAPs) or using them in miniaturized robotic devices is favorable considering their low actuation potential and high power-to-weight ratio. ${ }^{16-18} \mathrm{CP}$ microactuators are conventionally manufactured through a combination of chemical polymer fabrication methods and physical patterning techniques to define the device structures. ${ }^{19-22}$ Processing microactuators using microfabrication techniques such as photolithography is complicated, time consuming, and makes it difficult to integrate the actuators with current technologies. Polymer fabrication includes a broad range of crosslinking or polymerization methods such as photocrosslinking (ultraviolet [UV] curing), vapor phase polymerization, or electropolymerization. These techniques, although offering precise definition and patterning, allow fabrication by patterning a planar geometry in two dimensions ( $x$ and $y$ ) and allow only a limited fabrication in the third dimension $(z)$ by stacking and patterning consecutive layers, so called $2.5 \mathrm{D}$ fabrication. Additive manufacturing techniques such as $3 \mathrm{D}$ printing solve this problem by allowing full 3D control over the pattern definition. It is also a very promising technique owing to its ability to print or define the structures without using any harsh physical conditions or aggressive chemical agents. Polymers being sensitive to extreme physical conditions and reactive chemical agents will especially benefit from the rapidly growing field of additive manufacturing. The method offers the potential to reduce costs and increase the speed of prototyping for rapid product evaluation.

The current extrusion 3D printing of soft robotic devices is limited to the millimeter scale only, with the minimum dimension of the printed actuating structures reported over $200 \mu \mathrm{m}$. In this work we explore the possibilities of 3D printing to fabricate microactuators and explore the dimensional limits possible with extrusion-based 3D printing. Using a custom-built 3D printer, we fabricated microrobots comprising soft actuators in the sub- $100 \mu \mathrm{m}$ range. To extend the capabilities of extrusion 3D printing, we develop the printing process to pattern the electrical layer to fabricate microrobots with multiple microactuators that can be individually controlled. The successful $3 \mathrm{D}$ printing of soft microactuators provides the foundation to exclusively print microactuators with different device designs to exploit the ease and versatility of $3 \mathrm{D}$ printing process.

\section{Materials and Methods}

\section{Materials}

UV curable Ebecryl 4491, an aliphatic urethane acrylate gel, was purchased from Allnex Belgium. Pyrrole was purchased from Sigma-Aldrich and was distilled and stored at $-20^{\circ} \mathrm{C}$ before use. Sodium dodecylbenzenesulfonate (NaDBS) was acquired from Sigma-Aldrich and used as received. As the substrate for $3 \mathrm{D}$ printing, standard microscopic glass slides were used. For electropolymerization and electrochemical characterizations in an electrochemical cell, a titanium mesh was used as the counter electrode. The silver/silver chloride $(\mathrm{Ag} / \mathrm{AgCl})$ reference electrode used (model RE-5B) was purchased from BASi, and all the electric potentials measured were versus $\mathrm{Ag} / \mathrm{AgCl}$.

\section{D printer}

For 3D printing microactuators we used a custom-built extrusion $3 \mathrm{D}$ printer. $^{23}$ The basis of the $3 \mathrm{D}$ printer is a threeaxis ( $x-y-z$ axis) programmable CNC milling stage (Sherline 8020) complemented with a high-precision fluid dispensing system (Ultimus; Nordson EFD) (Fig. 1). A Luer lock syringe $(5 \mathrm{~mL})$, actuated by the dispensing system and fitted with tapered tip (Gauge 27, ID $0.20 \mathrm{~mm}$; Nordson EFD), was used to dispense gel. The rate of extrusion (printing) was controlled by the lateral motion of the stage $(\sim 2.5 \mathrm{~mm} / \mathrm{s})$ along with the dispensing air pressure (50-60 psi).

\section{Device design}

The process of $3 \mathrm{D}$ printing starts with a computer-aided design model or software design of the structure or device that needs to be printed. We programmed the design directly in g-code using LinuxCNC/AXIS software (version 2.5.0) to design the structure of microactuators. The g-code directly instructed the $3 \mathrm{D}$ printer to move along the three directions $(x-y-z$ axis) with intended position and speed.

\section{$3 D$ printing soft microactuator structure}

The starting substrate was a thin layer of gold $(40 \mathrm{~nm})$ sputtered (Dynavac Magnetron) over a cleaned glass slide to

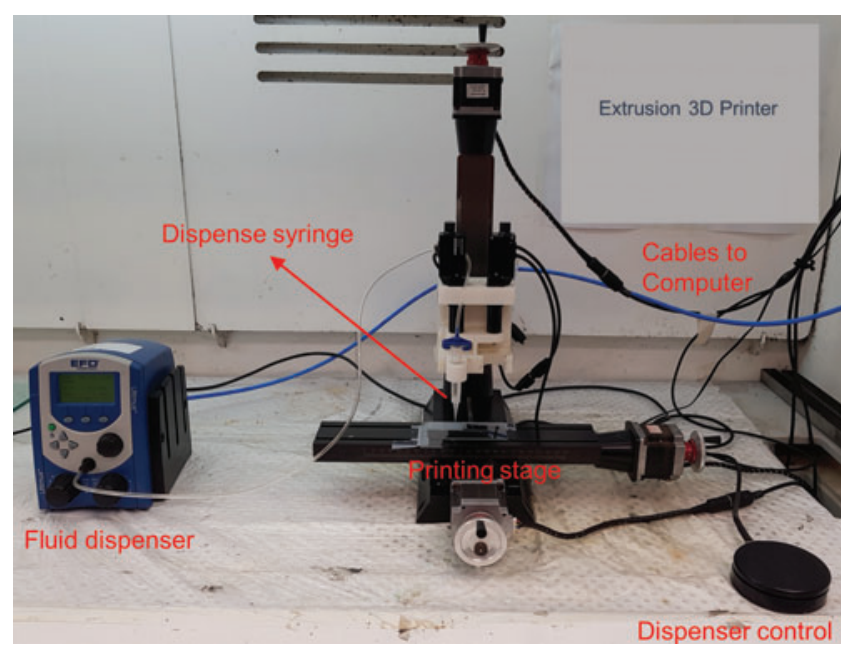

FIG. 1. Photograph of the custom-built extrusion 3D printer. 3D, three-dimensional. Color images are available online. 
form a conductive layer. A single layer of UV curable gel was printed using a custom extrusion $3 \mathrm{D}$ printer to form the body and arms of the microactuator. Subsequently, additional layers were printed over the body to control rigidity of different parts of the actuator, allowing for the controlled motion of the more flexible arms. Afterward, the printed structure was cured under a UV source of intensity $19 \mathrm{~W} / \mathrm{cm}^{2}$ for $60 \mathrm{~s}$ (Dymax BlueWave 75).

\section{Preparing polymer microactuators}

The printed and cured gel along with the attached gold was peeled off from the glass slide exploiting the weak adhesion between gold and glass surface. ${ }^{24}$ Finally, a layer of $\mathrm{CP}$ polypyrrole was deposited on the gold layer through electropolymerization in an aqueous ionic solution of $0.1 \mathrm{M}$ NaDBS containing $0.1 \mathrm{M}$ pyrrole monomer. The thickness of the polymer layer, which acts as the active layer, could be controlled by manipulating the electropolymerization conditions (mainly time). Electropolymerization was carried out in a three-electrode (working, counter, and reference) electrochemical cell using a potentiostat (Model 363; Princeton Applied Research) to apply an oxidizing potential of $0.65 \mathrm{~V}$ for different durations at room temperature. Figure 2 shows the flow of the fabrication steps involved in the manufacturing of microactuators using $3 \mathrm{D}$ printing.

A Top view

1

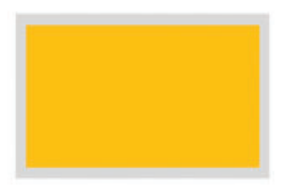

2

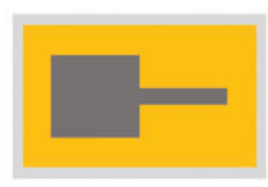

3

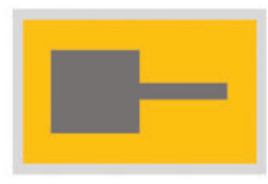

4

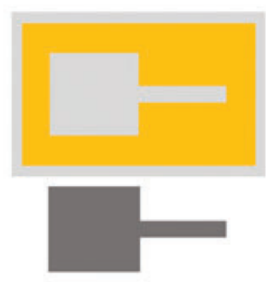

5

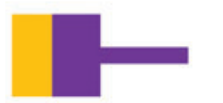

Side view
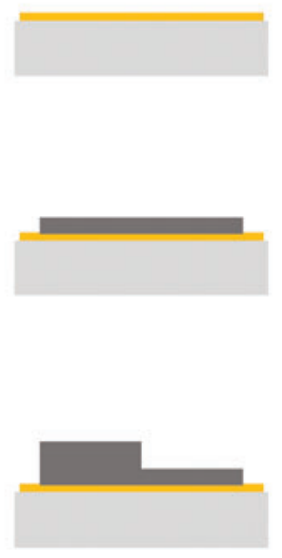

C
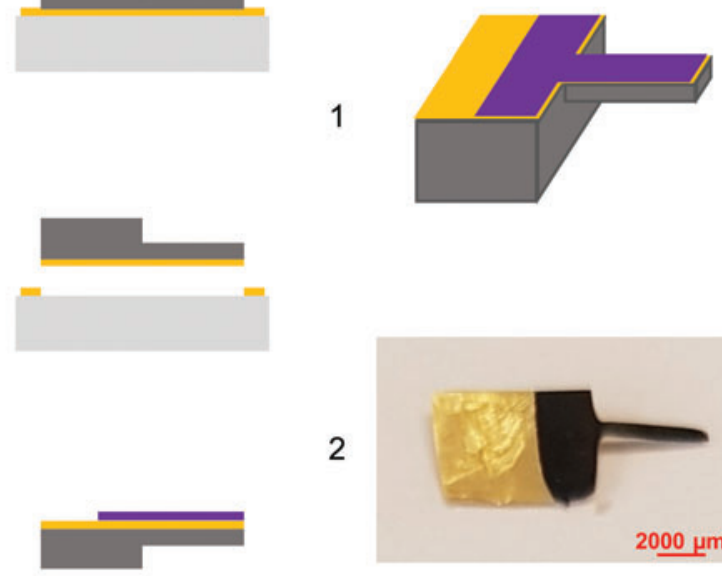

2

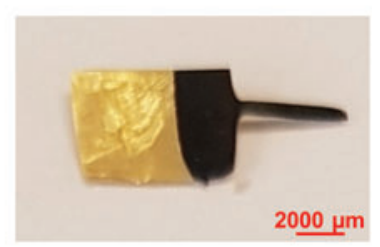

FIG. 2. (A) Schematic diagram illustrating the fabrication steps: (1) evaporate $\mathrm{Au}$ on glass substrate; (2) extrusion print first structural layer; (3) print consecutive layers to form rigid body; (4) remove the printed structure by lift-up; and (5) deposit polypyrrole through electropolymerization. (B) Electropolymerization of the printed structure in an electrochemical cell with WE, RE, and CE. (C) (1) 3D sketch of the final microactuator; and (2) photograph of the fabricated microactuator. CE, counter electrode; RE, reference electrode; WE, working electrode. Color images are available online. 

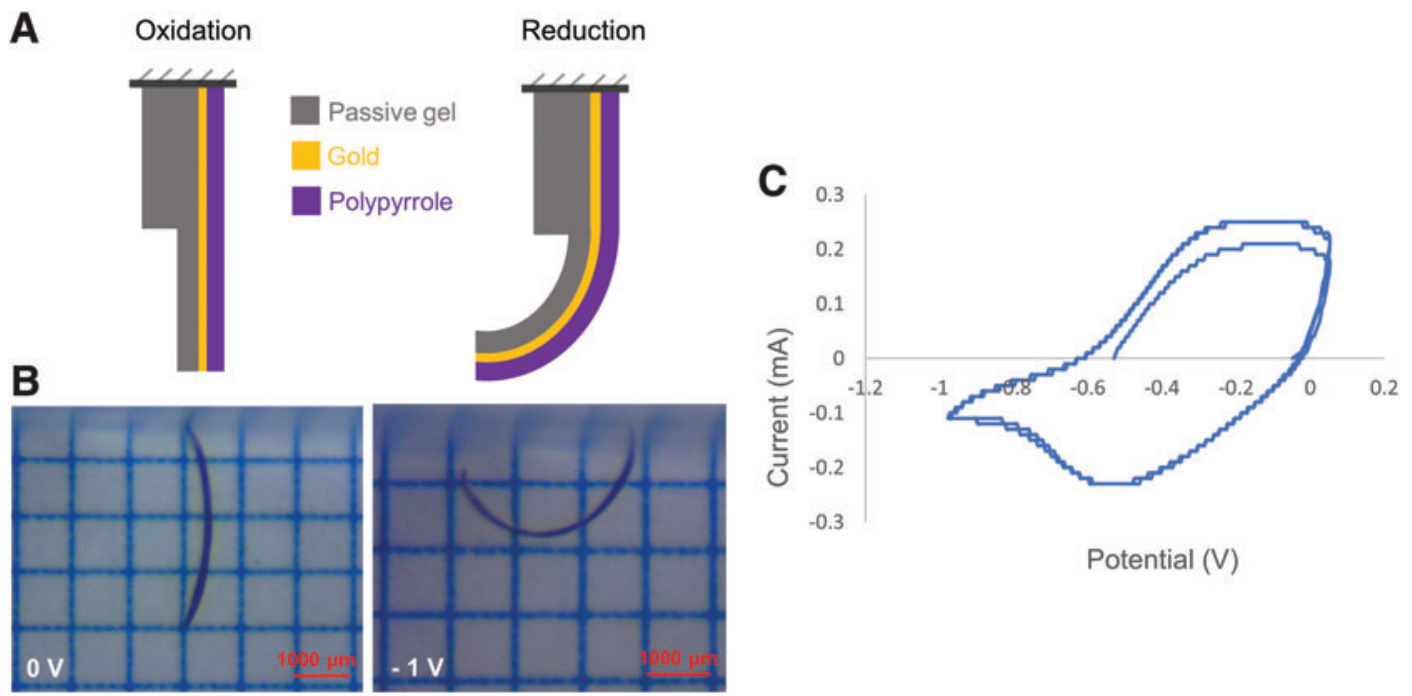

FIG. 3. Electrical actuation of the microactuator undergoing a redox state change. (A) Sketch to illustrate the orientation of the microactuator with different layers. (B) Motion of the 3D printed microactuator under an applied potential. (C) CV of the $3 \mathrm{D}$ printed polymer microactuator. $\mathrm{CV}$, cyclic voltammogram. Color images are available online.

NaDBS. As depicted in Figure 3, the microactuator moves normally as a unimorph bending PPy-microactuator, with fast switching between the two states ${ }^{25,26}$; and the corresponding cyclic voltammogram shows the PPy redox peaks at their usual potentials (approximately -0.5 and $-0.2 \mathrm{~V}$ for the reduction and oxidation, respectively). To compare the actuation motion of different microactuators, we calculated the displacement for the tip of the microactuator. Upon electrochemical reduction the PPy layer expanded leading toward the bending of microactuator and the tip moved $3500 \mu \mathrm{m}$ in $30 \mathrm{~s}$. This is comparable to the performance of the soft microactuators fabricated with conventional microfabrication techniques. ${ }^{27} \mathrm{Soft}$ microactuators of the dimension $200 \times 50 \times 48 \mu \mathrm{m}^{3}$ fabricated with electrodeposition and laser ablation technique reported a tip displacement of $95 \mu \mathrm{m}$ at $\pm 0.6 \mathrm{~V}, 0.005 \mathrm{~Hz}^{28}$ Fabricated with similar classical methods, EAP microactuators of the dimension $580 \times 220 \times 160 \mu \mathrm{m}^{3}$ and $5000 \times 1000 \times 48 \mu \mathrm{m}^{3}$ were reported to produce a tip displacement of $125 \mu \mathrm{m}$ at $\pm 1 \mathrm{~V}$ and $2100 \mu \mathrm{m}$ at $\pm 0.5 \mathrm{~V}$, respectively. ${ }^{21,29}$ Electronic $\mathrm{CP}$ actuators of the size $900 \times 300 \times 17 \mu \mathrm{m}^{3}$, fabricated with spin coating and dry etching methods, produced displacement of $950 \mu \mathrm{m}$ at $\pm 2 \mathrm{~V}, 0.05 \mathrm{~Hz}^{30}$ Using a bottom-up fabrication approach, $\mathrm{CP}$ actuators of the size $20 \times 5 \times 0.07 \mathrm{~mm}^{3}$ were manufactured, reporting a displacement of $170 \mu \mathrm{m}$ at $\pm 1 \mathrm{~V}$, $0.1 \mathrm{~Hz}^{31}$ On the contrary fabricated with top-down microfabrication approach, EAP microactuators of the size $650 \times 100 \times 10 \mu \mathrm{m}^{3}$ reported $127 \mu \mathrm{m}$ displacement at $\pm 4 \mathrm{~V}$, $0.2 \mathrm{~Hz}^{32}$
FIG. 4. Frequency versus displacement variation for the microactuators with different electropolymerization time. Color images are available online.

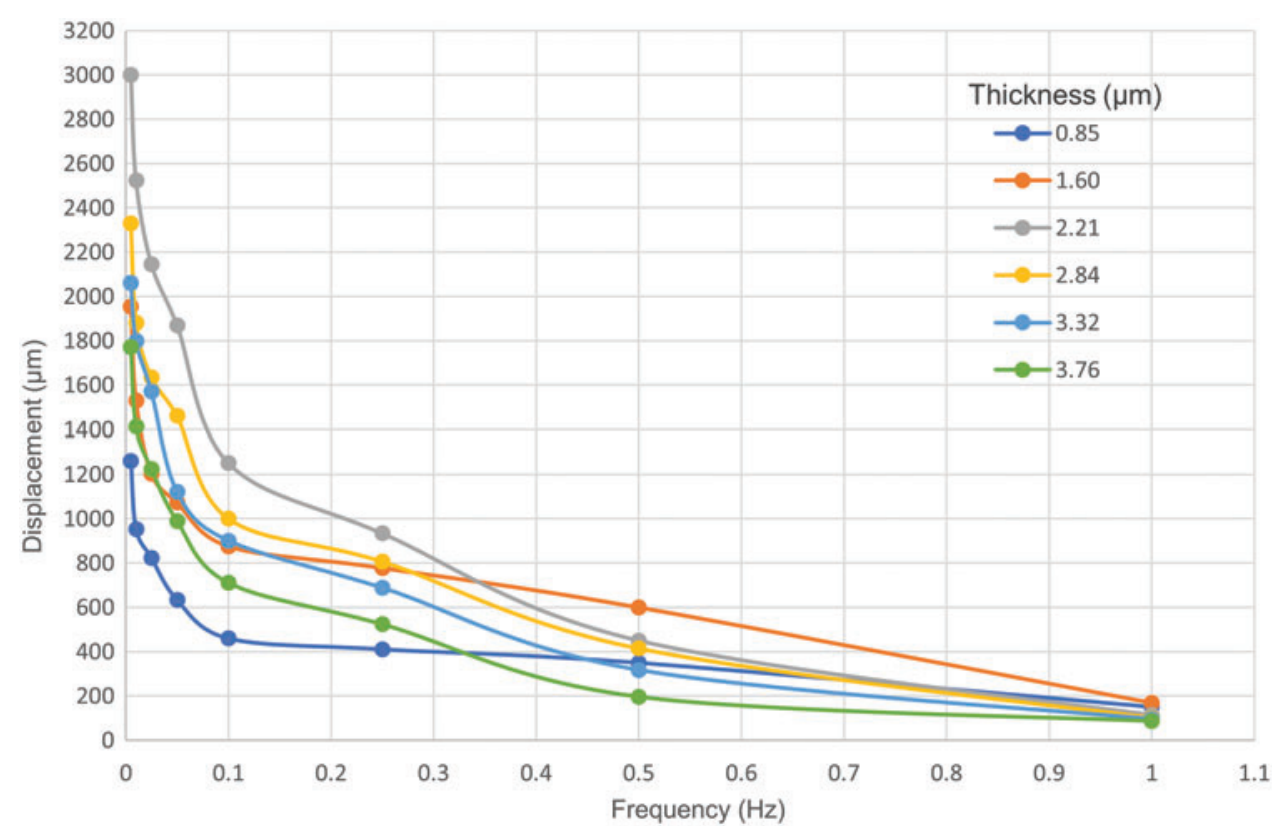


Table 1. Table Listing the Duration of EleCtropolymerization USED FOR DEPOSITING The CoRresponding Thicknesses of Polypyrrole

\begin{tabular}{lllllll}
\hline $\begin{array}{c}\text { Electropolymerization } \\
\text { time (minutes) }\end{array}$ & 10 & 15 & 20 & 25 & 30 \\
$\begin{array}{c}\text { Thickness of } \\
\text { polypyrrole }(\mu \mathrm{m})\end{array}$ & 0.85 & 1.60 & 2.21 & 2.84 & 3.32 & 3.76 \\
\hline
\end{tabular}

\section{Effect of polypyrrole thickness on actuation motion}

After the successful actuation of the 3D printed microactuator, we wanted to study the effect of the polypyrrole thickness, which is controlled by the electropolymerization time, on the bending motion of the microactuators. To analyze this, we fabricated microactuators with varying polypyrrole thickness and operated them at different frequencies to observe the displacement during actuation (Fig. 4). The microactuators $3 \mathrm{D}$ printed for this study were $300 \mu \mathrm{m}$ wide, $4000 \mu \mathrm{m}$ long, and $25 \mu \mathrm{m}$ thick, whereas the rigid body was $3000 \mu \mathrm{m}$ wide, $5000 \mu \mathrm{m}$ long, and $350 \mu \mathrm{m}$ thick. Figure 4 shows the variation of displacement of the microactuators with the frequency of operation, for microactuators with varying polypyrrole thicknesses fabricated with different electropolymerization times (Table 1). As observed for each sample, the displacement of the microactuators increased with the lower frequency of operation (or increased time of actuation). This behavior is typical of CP actuators and is attributed to the time the cations take to move into, or out of, the polypyrrole matrix, which causes the swelling of the polymer resulting in a bending motion. ${ }^{33,34}$ The lower the frequency of actuation, the longer time available for the microactuator to reduce or oxidize, resulting in more ingress or egress of ions from the polypyrrole matrix. The increased number of ions in and out of the polymer matrix thus produced larger displacement, and the trend was consistent for all the 3D printed microactuators independent of the thickness of polypyrrole (Fig. 4).
Furthermore, the thickness of polypyrrole not only impacts the displacement of an actuator positively due to the force generated by the volume change but also impacts the actuation negatively due to the fact that the layer has a bending stiffness. ${ }^{29}$ As noticed in Figure 4, the microactuators with thinner polypyrrole displaced (bent) more than the thicker ones at higher frequency of operation. This behavior can be understood from the fact that the displacement of a bending beam, with moment of inertia $I$ and Young's modulus $E$, is inversely proportional to the bending stiffness $I \cdot E$ of the beam which scales with the thickness cubed. ${ }^{27,35}$ A thicker polypyrrole would, therefore, not only generate more force but also oppose the bending motion due to the increased stiffness. At higher frequency, the duration of actuation is not long enough for the ions to diffuse completely into the polymer matrix. In such scenario when the maximum insertion of ions is not reached, the negative obstructive effect due to the thickness of polypyrrole dominates the positive driving force. This interplay between the opposing parameters associated with the thickness of the microactuator determines the extent of bending motion it experiences at a given frequency of actuation. ${ }^{36,37}$ To validate this effect and estimate the optimum thickness of polypyrrole, we analyzed the displacement of microactuator against the thickness of polypyrrole at a selected frequency of actuation $(0.025 \mathrm{~Hz})$ (Fig. 5). Evidently, the microactuator with PPy thickness of $2.21 \mu \mathrm{m}$ displaced most at this actuation frequency. This is the case for all actuation frequencies; for example, in Figure 4, the microactuator with PPy thickness of $1.60 \mu \mathrm{m}$ displaced most at actuation frequency of $0.5 \mathrm{~Hz}$.

\section{Scalability of the $3 D$ printing process}

Next, we wanted to explore the limits of the 3D printing process to manufacture miniaturized soft actuators. We were interested in analyzing how small the microactuators can be printed using the custom-built 3D printer and still be actuated by normal manual handling. For this purpose, we fabricated microactuators with arm lengths ranging from 1000 up to

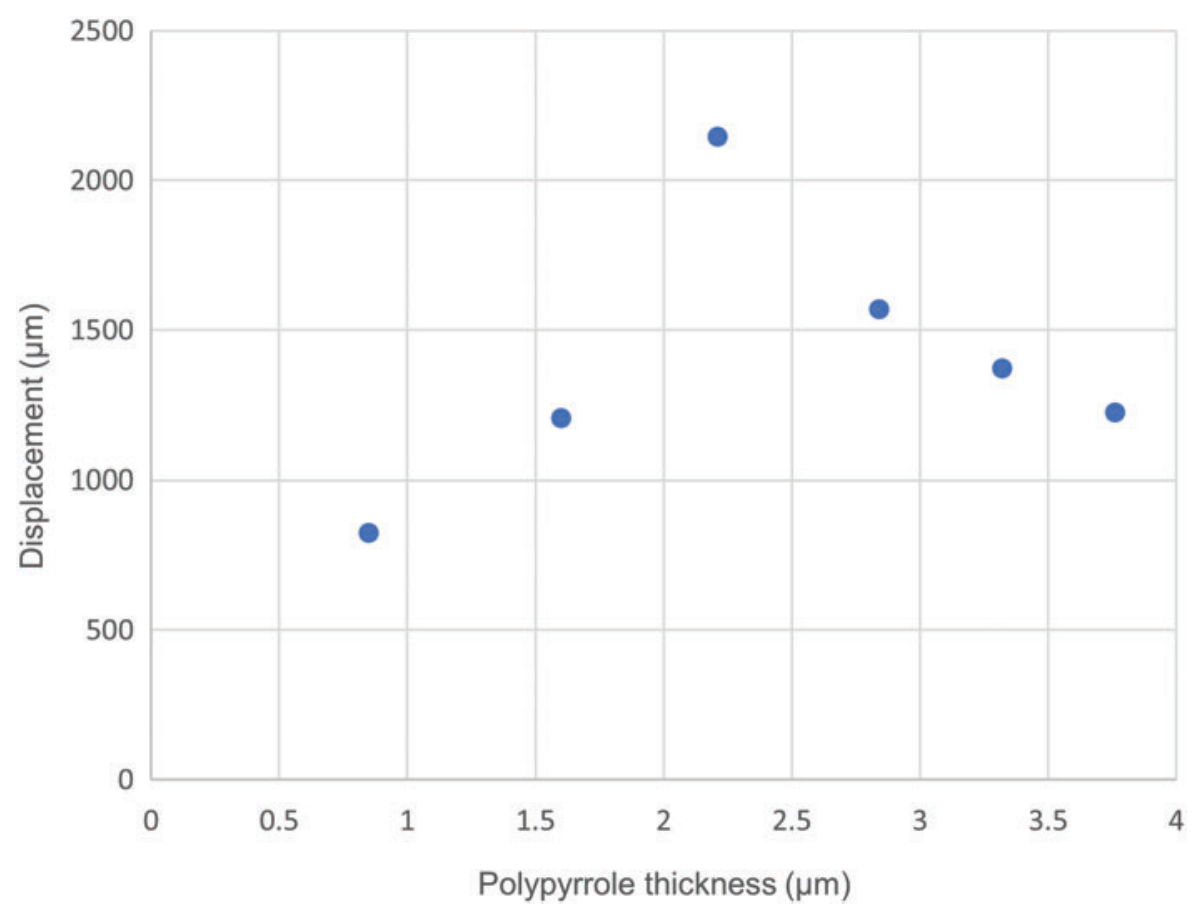

FIG. 5. Polypyrrole thickness versus displacement variation for the microactuators electrically actuated at $0.025 \mathrm{~Hz}$ frequency. Color images are available online. 
A

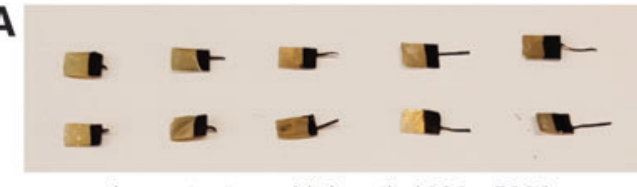

microactuators with length $1000-5000 \mu \mathrm{m}$
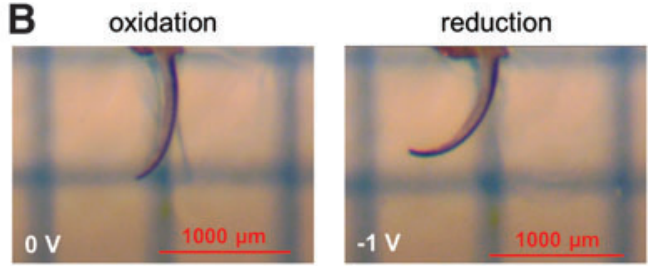

electrical actuation

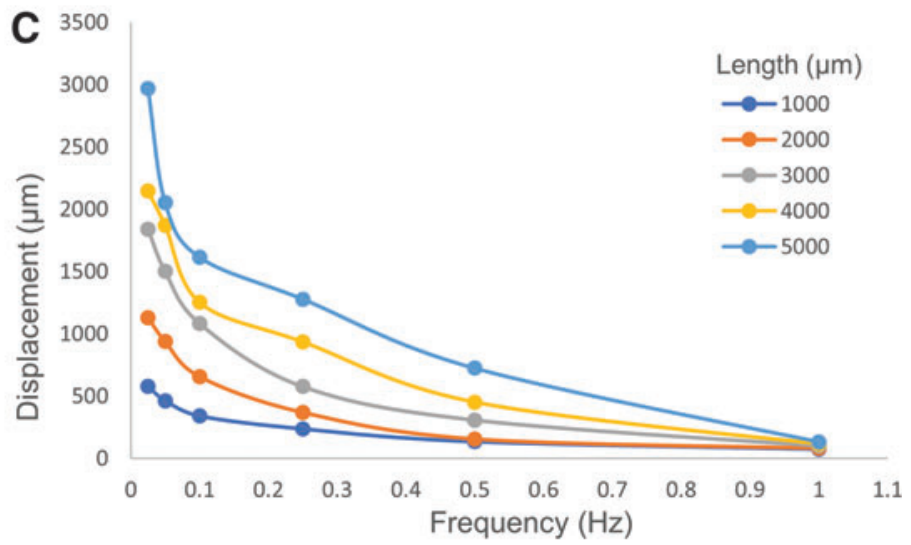

FIG. 6. (A) $3 \mathrm{D}$ printed microactuators with different lengths. (B) Electrical actuation of the smallest microactuator (1000 $\mu$ m). (C) Frequency versus displacement variation for the microactuators with different lengths. Color images are available online.

$5000 \mu \mathrm{m}$, with similar width and thickness $(300$ and $20 \mu \mathrm{m}$, respectively), and the rigid body of $3000 \mu \mathrm{m}$ wide, $5000 \mu \mathrm{m}$ long, and $350 \mu \mathrm{m}$ thick (Fig. 6A). We then actuated these actuators at different frequencies to examine the bending motion in terms of the displacement of the tip; Figure 6B shows the actuation of the smallest $3 \mathrm{D}$ printed microactuator. As expected, the microactuators with longer lengths displaced more, with a consistent trend throughout the frequency of actuation (Fig. 6C). This is expected from bending beam actuators where the displacement of the tip is proportional to the length of the microactuator. ${ }^{35,37}$

FIG. 7. (A) Schematic diagram showing the process flow to fabricate individually controlled microactuators: (1) evaporate $\mathrm{Au}$ on glass slide; (2) print the pattern layer; (3) remove pattern layer with lift-up to leave patterned Au-coated substrate; (4) print the complete structural layer; (5a) remove the structure with lift-up; (5b) the structure after removal from top side; $(5 \mathrm{c})$ the structure from bottom side; and (6) electropolymerize to deposit polypyrrole forming the microactuator device. (B) 3D sketch of the individually controlled microactuator. (C) Individually controlled microactuator fabricated using patterning by printing. Color images are available online.
A

1

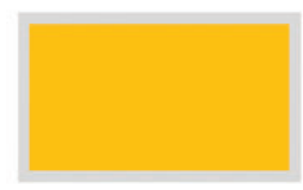

2

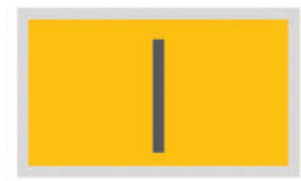

3

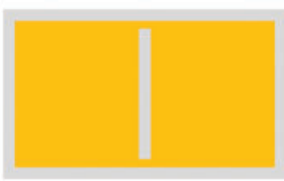

4

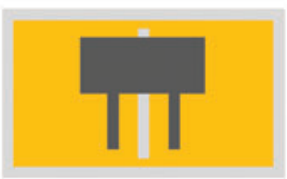

The smallest of these microactuators was fabricated using a double pass (two lines) of the 3D printer to form the actuator arm (Fig. 6B). In this process the printer deposited the passive gel in a continuous U-shaped manner instead of just terminating at the tip of the arm. The width of the line printed with an extrusion tip depends on several factors such as viscosity of gel, air pressure, feed rate, tip diameter, and the distance between the nozzle and printing platform. ${ }^{38}$ With our extrusion 3D printer (Fig. 1), we obtained a line width of $200 \mu \mathrm{m}$ comparable to the tip diameter, while the second adjacent (parallel) line was printed at half line width from the 
A front view before actuation

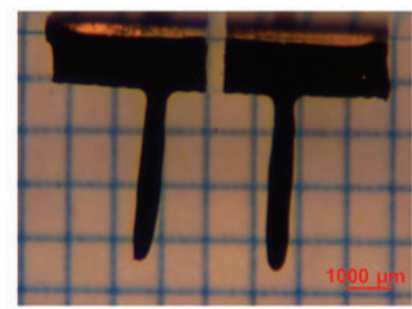

Actuators $400 \times 3000 \times 25 \mu \mathrm{m}^{3}$ Body $6000 \times 5000 \times 350 \mu \mathrm{m}^{3}$
B

oblique view during actuation

1
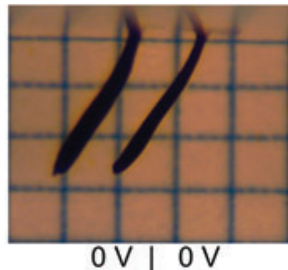

2

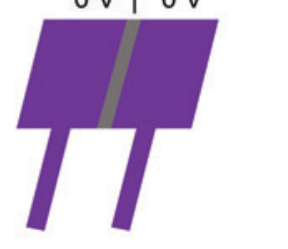

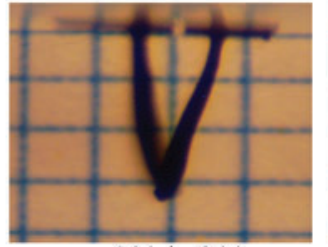

$-1 \mathrm{~V} \mid 0 \mathrm{~V}$

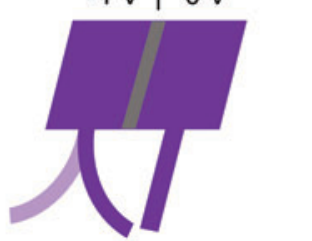

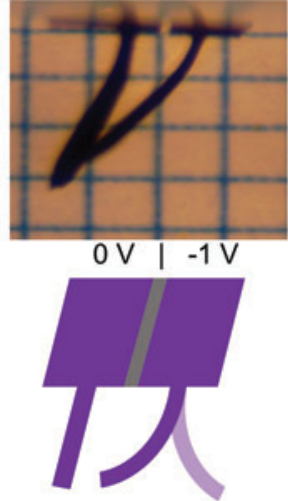

FIG. 8. (A) Front view of the individually controlled microactuator before electrical actuation in aqueous ionic solution. (B) (1) Independent electrical actuation of the individual microactuators attached with the same body; and (2) schematic illustration of the individually controlled microactuators showing the independent movement during selective actuation. Color images are available online.

first line to achieve a reasonable uniform thickness of the resulting structure. Although we could have scaled down even further by $3 \mathrm{D}$ printing microactuators with just one pass of the printer (one line), we determined that better quality prints were achieved by continuous printing. It was observed that terminating the printing process (gel extrusion) at the microactuator arm tip would often result in deposition of unwanted drop of passive gel, which would then require additional treatment (cutting) afterward to finalize the desired shape. Furthermore, the minimum thickness of the first gel layer was $20 \mu \mathrm{m}$ because we observed bending (and occasional breakage) of the fragile microactuator while lifting off from the substrate for actuators thinner than $20 \mu \mathrm{m}$. To avoid back-processing after the printing process and $3 \mathrm{D}$ print the complete structure as much as possible, we decided to print the microactuator arms with two continuous lines of gel extrusion.

\section{Patterning by printing}

$3 \mathrm{D}$ printing provides the flexibility to print a variety of designs, so we developed a simple process of patterning the printed structures to manufacture more complicated devices involving multiple actuating parts. Complex devices comprising of multiple actuators are typically fabricated through conventional microfabrication techniques (such as photolithography) to pattern the electrically conductive (gold) layer, allowing for individual actuator control. ${ }^{39}$ To achieve such patterning with $3 \mathrm{D}$ printing, we modified our $3 \mathrm{D}$
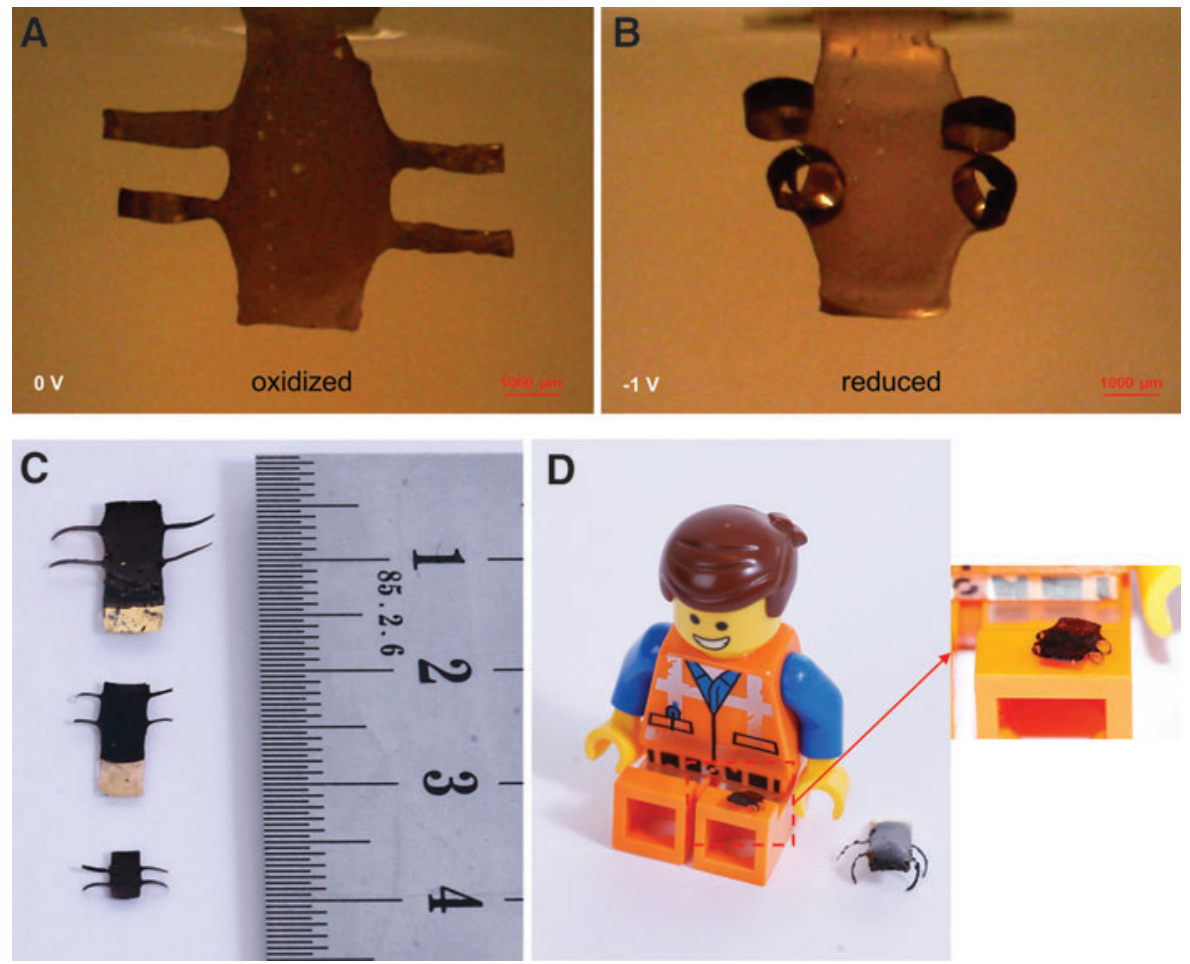

D

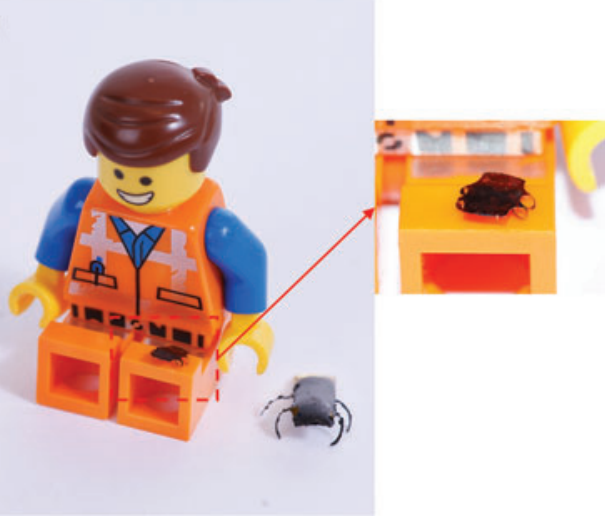

FIG. 9. Microrobotic devices with varied dimensions depicting the scale of fabrication. (A, B) Electrical actuation of a microrobotic structure with four flexible moving arms/legs and a rigid body. The fingers are $300 \mu \mathrm{m}$ wide and $2000 \mu \mathrm{m}$ long. The body is $2000 \times 5000 \mu \mathrm{m}^{2}$ and is multiple layers thick. (C) Three microrobotic actuators placed in oxidized state along a millimeter scale. (D) The smallest (in inset) and the largest microrobot placed in reduced state along with a LEGO minifigure. Color images are available online. 
printing process to pattern the electrically conducting layer to create electrically isolated electrodes, thereby allowing the fabrication of individually controlled microactuators for independent control and actuation. Since we wanted to avoid any etching steps and use 3D printing as much as possible, we developed a simple and effective method of patterning the gold layer through 3D printing (Fig. 7A). We first printed a pattern layer over the Au-coated glass substrate and then physically removed this printed layer. This "lift-up" patterning process removed Au from the glass and left behind the desired patterned $\mathrm{Au}$ coated substrate. ${ }^{40}$ This extra printing step allows for patterning of the electrically conducting layer, thereby enhancing the versatility of the process to fabricate intricate complex structures without the need of any additional etching techniques. This novel patterning process not only simplified the process of device design but also helps avoid the use of harsh chemical and physical methods that could be harmful to device materials such as CPs.

Figure 7A illustrates the process flow for patterning the conductive layer using 3D printing. In this example, we patterned and fabricated two individually controlled microactuators on a monolithic body for independent actuation (Fig. 7B), demonstrating the printing of complicated design structures. Figure 7C shows the finished device having two individually controlled microactuator arms of width $400 \mu \mathrm{m}$, length $3000 \mu \mathrm{m}$, and thickness $25 \mu \mathrm{m}$. The two arms were attached to a single body of width $6000 \mu \mathrm{m}$, length $5000 \mu \mathrm{m}$, and thickness $350 \mu \mathrm{m}$. To demonstrate these independently controlled microactuators, we individually actuated the microactuator arms as illustrated in Figure 8. As observed, the two microactuator arms attached to the single body moved (bent) individually corresponding with the electrical actuation applied (Fig. 8B).

\section{$3 D$ printing microrobots}

To demonstrate the scalability and explore the applications of our developed 3D printing process, we fabricated microrobotic structures with multiple arms/legs and of varying dimensions. We 3D printed microrobots with four microactuator arms that actuate at a low operating potential of 0 to $-1 \mathrm{~V}$. Figure $9 \mathrm{C}$ shows some of these 3D printed microrobots $\left(6 \times 4.5 \mathrm{~mm}^{2}, 7 \times 9.5 \mathrm{~mm}^{2}\right.$, and $\left.14 \times 12 \mathrm{~mm}^{2}\right)$ compared to a common ruler and Figure 9D compared to a LEGO minifigure to showcase their relative dimensions. Figure $9 \mathrm{~A}$ and $\mathrm{B}$ shows the activation of such a microrobot. The four flexible actuating arms/legs were $300 \mu \mathrm{m}$ wide, $2000 \mu \mathrm{m}$ long, and $25 \mu \mathrm{m}$ thick, whereas the rigid body they were connected to was $2000 \mu \mathrm{m}$ wide, $5000 \mu \mathrm{m}$ long, and $350 \mu \mathrm{m}$ thick. The microrobot arms/legs show the typical bending of the PPy microactuators. These microrobots can potentially be used to grasp and release objects or as microwalkers. To enable in air operation, the microactuators have to be combined with ionogels, ${ }^{41,42}$ or other solid-state ion sources into a so called trilayer actuator, ${ }^{21,27,28}$ which is ongoing work.

\section{Conclusions}

Manufacturing microactuators using 3D printing provides a smooth design-to-product process, allowing for the fabrication of microrobots with ease. We have developed a 3D printing process that enables fabrication of microactuators and microrobots with varied structures and dimensions down to the micrometer domain. Using a custom-built extrusion $3 \mathrm{D}$ printer, we successfully scaled down the actuators to dimension of $300 \times 1000 \mu \mathrm{m}^{2}$ and thickness of $20 \mu \mathrm{m}$. The smallest 3D printed actuator/robot showed improved actuation motion (bending) at low operating potentials $(0$ to $-1 \mathrm{~V})$. The 3D printed microactuators were operated in the frequency range of 0.005 to $1.0 \mathrm{~Hz}$, achieving displacements from 100 to $3000 \mu \mathrm{m}$. The resulting performance of the $3 \mathrm{D}$ printed microactuators was similar to those fabricated using conventional methods such as photolithography and laser ablation. ${ }^{21,27-32}$

To extend the adaptability of the $3 \mathrm{D}$ printing process, we developed a new patterning process to define the conductive layer using just one extra step of printing to transfer the design. The new method of "patterning by printing" using lift-up is a simple and promising solution for patterning the structures, which would be favorable for polymers that are sensitive to extreme processing conditions. Using this new patterning method, we demonstrated individual control of microactuators by independently actuating different flexible arms attached to the same microrobot body.

To demonstrate the scalability and versatility of our 3D printing process, we fabricated microrobots with multiple actuating arms (2-4) and dimensions ranging from $6 \times 4.5$ to $14 \times 12 \mathrm{~mm}^{2}$, providing an understanding of the limits to microfabricate microactuators and microrobots using 3D printing. The presented new processes have pushed the limits of $3 \mathrm{D}$ printing into the micrometer domain enabling the design and fabrication of a plethora of (micro-) devices with independently controlled microactuators, to potentially achieve complex capabilities. This will broaden the purview of soft robotics with new generations of $3 \mathrm{D}$ printed microrobots.

\section{Authors' Contributions}

M.T., G.M.S., and E.W.H.J. designed the experiments and wrote the article. M.T. and E.W.H.J. performed the experiments.

\section{Author Disclosure Statement}

No competing financial interests exist.

\section{Funding Information}

We thank the EU Marie Sklodowska-Curie Actions Initial Training Network MICACT (641822), the Swedish Research Council (2014-3079), and the University of Wollongong's Visiting International Scholar Award and Global Challenges Program for their financial support. We thank Prof. Marc in het Panhuis and Dr. Holly Warren for their support in the laboratory.

\section{References}

1. Wallin TJ, Pikul J, Shepherd RF. 3D printing of soft robotic systems. Nat Rev Mater 2018;3:84-100.

2. Zolfagharian A, Kouzani AZ, Khoo SY, et al. Evolution of 3D printed soft actuators. Sensors Actuators A Phys 2016; 250:258-272.

3. Haghiashtiani G, Habtour E, Park S-H, et al. 3D printed electrically-driven soft actuators. Extrem Mech Lett 2018; 21:211-218.

4. Gonzalez D, Newell B, Garcia J, et al. 3-D printing of dielectric electroactive polymer actuators and characterization of dielectric flexible materials. In: Proceedings of the ASME 2018 Conference on Smart Materials, Adaptive Structures and Intelligent Systems. San Antonio, TX: ASME, 2018, pp. SMASIS2018-8011. 
5. Schaffner M, Faber JA, Pianegonda L, et al. 3D printing of robotic soft actuators with programmable bioinspired architectures. Nat Commun 2018;9:878.

6. Truby RL, Wehner M, Grosskopf AK, et al. Soft somatosensitive actuators via embedded 3D printing. Adv Mater 2018;30:1706383.

7. Zhang Y-F, Zhang $\mathrm{N}$, Hingorani $\mathrm{H}$, et al. Fast-response, stiffness-tunable soft actuator by hybrid multimaterial 3D printing. Adv Funct Mater 2019;29:1806698.

8. Carrico JD, Traeden NW, Aureli M, et al. Fused filament 3D printing of ionic polymer-metal composites (IPMCs). Smart Mater Struct 2015;24:125021.

9. Carrico JD, Hermans T, Kim KJ, et al. 3D-printing and machine learning control of soft ionic polymer-metal composite actuators. Sci Rep 2019;9:17482.

10. Zhang Z, Demir KG, Gu GX. Developments in 4Dprinting: a review on current smart materials, technologies, and applications. Int J Smart Nano Mater 2019;10:205-224.

11. Wu J-J, Huang L-M, Zhao Q, et al. 4D printing: history and recent progress. Chinese J Polym Sci 2018;36:563-575.

12. López-Valdeolivas M, Liu D, Broer DJ, et al. 4D printed actuators with soft-robotic functions. Macromol Rapid Commun 2018;39:1700710.

13. Shinoda H, Azukizawa S, Maeda K, et al. Bio-mimic motion of 3D-printed gel structures dispersed with magnetic particles. J Electrochem Soc 2019;166:B3235-B3239.

14. Bakarich SE, Gorkin III R, Panhuis $\mathrm{M}$ in het, et al. 4D printing with mechanically robust, thermally actuating hydrogels. Macromol Rapid Commun 2015;36:1211-1217.

15. Naficy S, Gately R, Gorkin III R, et al. 4D printing of reversible shape morphing hydrogel structures. Macromol Mater Eng 2017;302:1600212.

16. Smela E. Conjugated polymer actuators for biomedical applications. Adv Mater 2003;15:481-494.

17. Spinks GM, Liu L, Wallace GG, et al. Strain response from polypyrrole actuators under load. Adv Funct Mater 2002; 12:437-440.

18. Carpi F, Kornbluh R, Sommer-Larsen P, et al. Electroactive polymer actuators as artificial muscles: are they ready for bioinspired applications? Bioinspir Biomim 2011;6:45006.

19. Yang J, Zhang C, Wang X, et al. Development of micro- and nanorobotics: a review. Sci China Technol Sci 2019;62:1-20.

20. Smela E. Microfabrication of PPy microactuators and other conjugated polymer devices. J Micromech Microeng 1999; 9:1-18.

21. Gursel A, Valerie D, Philippe R, et al. Conducting polymer microactuators operating in air. J Micromech Microeng 2009; 19:25017.

22. Jager E, Krogh M. Method flayeredor producing a micromachined device. 2003, Patent WO2004092050.

23. Mire CA, Agrawal A, Wallace GG, et al. Inkjet and extrusion printing of conducting poly(3,4-ethylenedioxythiophene) tracks on and embedded in biopolymer materials. J Mater Chem 2011;21:2671-2678.

24. Smela E, Ingands O, Lundstrom I. Differential adhesion method for microstructure release: an alternative to the sacrificial layer. In: Proceedings of the International Solid-State Sensors and Actuators Conference-TRANSDUCERS'95, Stockholm, Sweden, June 25-29, 1995, Vol. 1, pp. 218-219.

25. Smela E, Inganäs O, Lundström I. Controlled folding of micrometer-size structures. Science (80-.) 1995;268:17351738.

26. Jager EWH, Smela E, Inganäs O. Microfabricating conjugated polymer actuators. Science (80-.) 2000;290:1540-1545.
27. Tyagi M, Pan J, Jager EWH. Novel fabrication of soft microactuators with morphological computing using soft lithography. Microsyst Nanoeng 2019;5:44.

28. Gaihre B, Alici G, Spinks GM, et al. Pushing the limits for microactuators based on electroactive polymers. J Microelectromechanical Syst 2012;21:574-585.

29. Gaihre B, Alici G, Spinks GM, et al. Synthesis and performance evaluation of thin film PPy-PVDF multilayer electroactive polymer actuators. Sensors Actuators A Phys 2011;165:321-328.

30. Khaldi A, Plesse C, Soyer C, et al. Conducting interpenetrating polymer network sized to fabricate microactuators. Appl Phys Lett 2011;98:164101.

31. Khaldi A, Falk D, Maziz A, et al. Fabrication and adhesion of conjugated polymer trilayer structures for soft, flexible micromanipulators. In: Proceedings of the SPIE 9798, Electroactive Polymer Actuators and Devices (EAPAD), April 15, 2016.

32. Maziz A, Plesse C, Soyer C, et al. Top-down approach for the direct synthesis, patterning, and operation of artificial micromuscles on flexible substrates. ACS Appl Mater Interfaces 2016;8:1559-1564.

33. Careem MA, Velmurugu Y, Skaarup S, et al. A voltammetry study on the diffusion of counter ions in polypyrrole films. J Power Sources 2006;159:210-214.

34. Zainudeen UL, Careem MA, Skaarup S. PEDOT and PPy conducting polymer bilayer and trilayer actuators. Sensors Actuators B Chem 2008;134:467-470.

35. Pei Q, Inganaes O. Electrochemical applications of the bending beam method. 1. Mass transport and volume changes in polypyrrole during redox. J Phys Chem 1992;96:10507-10514.

36. Bay L, Jacobsen T, Skaarup S, et al. Mechanism of actuation in conducting polymers: osmotic expansion. J Phys Chem B 2001;105:8492-8497.

37. Benslimane M, Gravesen P, West K, et al. Performance of polymer-based actuators: the three-layer model. In: Smart Structures and Materials 1999: Electroactive Polymer Actuators and Devices; International Society for Optics and Photonics, 1999, Vol. 3669, pp. 87-98.

38. He Y, Yang F, Zhao H, et al. Research on the printability of hydrogels in 3D bioprinting. Sci Rep 2016;6:29977.

39. Jager $\mathrm{EWH}$, Inganäs $\mathrm{O}$, Lundström I. Microrobots for micrometer-size objects in aqueous media: potential tools for single-cell manipulation. Science (80-.) 2000;288:2335.

40. Granlund T, Nyberg T, Stolz Roman L, et al. Patterning of polymer light-emitting diodes with soft lithography. Adv Mater 2000;12:269-273.

41. Zhong Y, Nguyen GTM, Plesse C, et al. Highly conductive, photolithographically patternable ionogels for flexible and stretchable electrochemical devices. ACS Appl Mater Interfaces 2018;10:21601-21611.

42. Zhong Y, Nguyen GTM, Plesse C, et al. Tailorable $\{\} ,3 \mathrm{D}$ structured and micro-patternable ionogels for flexible and stretchable electrochemical devices. J Mater Chem C 2019; 7:256-266.

Address correspondence to: Edwin W.H. Jager Sensor and Actuator Systems Department of Physics, Chemistry, and Biology (IFM) Linköping University Linköping 58183 Sweden

E-mail: edwin.jager@liu.se 